\title{
Erratum to: AVATAR Therapy for Refractory Auditory Hallucinations
}

\author{
Tom Craig, Tom Ward, and Mar Rus-Calafell
}

\section{Erratum to: \\ Chapter 4 in: B. Pradhan et al. (eds.), Brief Interventions for Psychosis, DOI 10.1007/978-3-319-30521-9_4}

Due to a mistake during processing this chapter was initially published with the copyright holder stated as "Springer International Publishing Switzerland".

This has now been corrected to the copyright holder "The Author(s)".

The updated online version of the original chapter can be found under DOI 10.1007/ 978-3-319-30521-9_4

T. Craig $(\bowtie) \bullet$ T. Ward $\bullet$ M. Rus-Calafell

Psychology and Systems Sciences, Kings College London, Institute of Psychiatry, Psychology and Neuroscience,

Box 33, HSPRD, De Crespigny Park, London SE5 8AF, UK

e-mail: thomas.craig@kcl.ac.uk 